\title{
A Comparison of Different Theoretical Models of Positron Lifetime Spectra for Polymers
}

\author{
J. KANSY* \\ Institute of Material Science, Silesian University \\ Bankowa 12, 40-007 Katowice, Poland
}

\begin{abstract}
The same reference positron lifetime spectra of polyurethane, measured in temperature range 112 to $390 \mathrm{~K}$, were analyzed with three theoretical models: the "conventional" model with three exponential decay component of discrete values of lifetimes, a model with one discrete exponential component and two "packages" of exponentials with log-normal distribution of annihilation rates, and a model considering positronium slow localization, positronium internal relaxation as well as "delayed" formation of positronium. It turns out that the two latter models fit to the experimental data with the same excellent quality, in spite of the fact that in both models the ratio of intensities related to para-positronium and ortho-positronium was constrained to be as 1:3.
\end{abstract}

PACS numbers: $78.70 . \mathrm{Bj}$

\section{Introduction}

Positrons injected into polymer form positronium (Ps) in its singlet (paraPs, $p$-Ps) or triplet state (ortho-Ps, o-Ps) with abundance ratio of 1:3 [1]. In vacuum the lifetimes of $p$-Ps and $o$-Ps are $0.125 \mathrm{~ns}$ and $142 \mathrm{~ns}$, respectively. In matter the $o$-Ps lifetime is radically reduced due to so called pick-off annihilation, i.e. annihilation of the positron from interior of Ps with an electron from the surrounding molecules, during a collision of Ps with a molecule. The reduction of $o$-Ps lifetime depends on density of matter around the particle. In polymers Ps is preferentially localized in the atomic-size free-volume holes, therefore the $o$-Ps lifetime, measured via positron annihilation lifetime spectroscopy (PALS) supplies valuable information of the mean hole sizes and their distribution [2-4].

The simplest analysis of the lifetime spectra makes use of its deconvolution into three exponential decay curves ascribed to $p$-Ps, unbound (free) positron $\left(\mathrm{e}^{+}\right)$

*e-mail: kansy@us.edu.pl 
and $o$-Ps annihilation processes. As a result, one obtains the spectrum parameters, lifetimes $\left(\tau_{i}\right)$ of positrons involved in a respective annihilation process and relative intensities $\left(I_{i}\right)$ of those processes. However, it turns out that some lifetime spectrum parameters resulting from the deconvolution are in strong disagreement with theoretical expectations. It is observed that the $p$-Ps lifetime $\tau_{1}$ is rather large with the expected value $\approx 0.125 \mathrm{~ns}$ and the lifetime component ratio for two species of Ps, $I_{1} / I_{3}$ is usually far from the theoretical value of $1 / 3$. It was shown $[5,6]$ that the discrepancies were artifacts resulting from not adequate theoretical model of spectrum used in analysis. According to the authors, both an o-Ps lifetime distribution and mainly an $\mathrm{e}^{+}$lifetime distribution (due to localization of $o$-Ps and $\mathrm{e}^{+}$in the free-volume holes of different shapes and sizes) influence the shape of lifetime spectrum. Therefore a discrete-term analysis overestimates the values of $\tau_{1}$ and $I_{1}$. This approach was applied successfully in many works [7-15] to analysis of lifetime spectra for different polymeric and other molecular substances.

Another explanation of the mentioned overestimations of $\tau_{1}$ and $I_{1} / I_{3}$ takes into account the processes of relaxation and localization of positronium [16-18]. Both processes are considered to be slow, of duration times comparable with $p$ Ps lifetime. As a result, lifetimes of $p$-Ps are elongated $\left(\tau_{1}>0.125 \mathrm{~ns}\right)$, because positronium can annihilate from non-relaxed states of low contact density between electron and positron. On the other hand, lifetimes of $o$-Ps are shortened as positronium can annihilate before its final localization. The latter decreases the relative intensity $\left(I_{3}\right)$ of the longest component of lifetime spectrum.

The aim of this paper is to compare results of analyses of the same series of polymer spectra carried out with help of discrete-component, distributedcomponent and slow relaxation and localization models. We are going to discuss not only the quality of fits of the theoretical models to experimental data but first of all values of the determined parameters and their physical meaning.

\section{Experimental and analysis}

A cross-linked polyurethane (PU) with molecular weight 1200 was investigated. It was synthesized in Dipartamento di Chimica Industriale e Ingegneria Chimica of Politecnico di Milano. For the investigated polyurethane the glass temperature $\left(T_{\mathrm{g}}\right)$ determined from differential scanning calorimetry (DSC) was $250 \mathrm{~K}$. Full details about its synthesis and about the measurement can be found in a previous paper [19].

The spectra were measured with conventional fast-fast spectrometer in situ in the temperature range from 114 to $390 \mathrm{~K}$ with step $\approx 10 \mathrm{~K}$. At each temperature three measurements were performed, each containing between 1.1 and $1.5 \mathrm{M}$ coincidence counts in a single spectrum. With help of special procedure for the time zero correction, the spectra were added to give a final spectrum of 4 to 4.5 $\mathrm{M}$ total counts. The signal-to-noise ratio was above 2000. A channel width was $0.0385 \mathrm{~ns}$. 
The experimental data were analyzed with LT program [20] in version 9 (additionally extended by a code for implementation of the slow relaxation and localization model). During the numerical analyses the same shape of the resolution function was fitted to all the measured spectra. It consists of a Gaussian of $\mathrm{FWHM}=0.224 \mathrm{~ns}(76 \%)$ and a Gaussian of FWHM $=0.340 \mathrm{~ns}(24 \%)$ shifted by 0.066 of channel. The estimated source correction was $10 \%$ of $0.386 \mathrm{~ns}$.

\section{Results and discussion}

\subsection{Three-discrete-component analysis}

The positron lifetime spectrum (disregarding the lifetime resolution) was described by a sum of three discrete components

$$
s(t)=\sum_{i=1}^{3}\left(I_{i} / \tau_{i}\right) \exp \left(-\left(t / \tau_{1}\right)\right) \quad \text { where } \quad \sum_{i=1}^{3} I_{i}=1,
$$

which, as it was already mentioned, was related to $p$-Ps, $\mathrm{e}^{+}$and $o$-Ps annihilations. By fitting Eq. (1) to the experimental spectra, values of 5 free parameters $\left(\tau_{1}, I_{2}, \tau_{2}, I_{3}, \tau_{3}\right)$ were determined.

The three-discrete-component analysis, similarly to huge amount of literature results for other polymers, gave values of $\tau_{1}$ and $I_{1} / I_{3}$ significantly far from the theoretical expectations (Fig. 1). Determined values of $\tau_{3}$ show typical temperature dependence observed for polymers transiting from glassy to elastic state (Fig. 2). Starting from low temperature up to the glass temperature $T_{\mathrm{g}}$ the dependence of $\tau_{3}$ on $T$ is weak. It becomes radically stronger above $T_{\mathrm{g}}$ and weakens again at so-called knee temperature $T_{\mathrm{k}}$ (in our case $T_{\mathrm{k}} \approx 340 \mathrm{~K}$ ). The $o$-Ps intensity $I_{3}$ (Fig. 3) increases almost linearly with $T$ up to $T_{\mathrm{g}}$. Just above $T_{\mathrm{g}}$ it bends a bit and next it continues the increase. The bend is often observed at $I_{3}$ at low temperatures and reflexes decrease in Ps formation above $T_{\mathrm{g}}$, because of release of radiation-induced electrons [21-23] from shallow electron traps in polymer structure.

The lifetime $\tau_{2}$ changes from 0.41 to $0.46 \mathrm{~ns}$ and its temperature dependence mirrors that for $\tau_{3}$ (not shown).

\subsection{Three-distributed-component analysis}

During the analysis the lifetime spectrum was expressed by

$$
s(t)=I_{1} \lambda_{1} \exp \left(-\lambda_{1} t\right)+\sum_{i=2}^{3} I_{i} \int_{0}^{\infty} \alpha_{i}(\lambda) \mathrm{d} \lambda \exp (-\lambda t)
$$

with $\sum_{i=1}^{3} I_{i}=1$ and $I_{1} / I_{3}=1 / 3$, where $\lambda_{1}=1 / \tau_{1}$ is the annihilation rate for $p$-Ps. According to suggestion given in papers [5-15], we assumed that first component of $s(t)$ is discrete. The rates for $\mathrm{e}^{+}$and $o$-Ps were assumed distributed and the distributions were described by $\alpha_{i}(\lambda)$.

As it was showed with help of simulated spectra, because of statistical errors, exact shape of $\alpha_{i}(\lambda)$ cannot be determined any way. There is a possibility to 


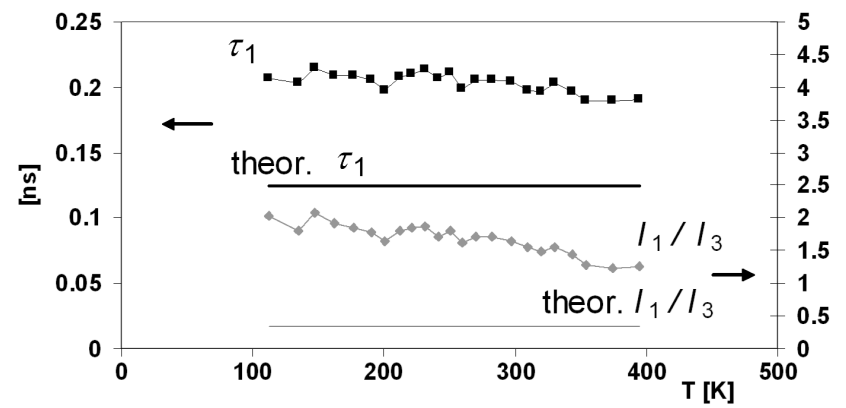

Fig. 1. The lifetime $\left(\tau_{1}\right)$ of the first component and the ratio of first and third component intensities $\left(I_{1} / I_{3}\right)$ determined in the three-discrete-component analysis for the PU spectra. The lines below represent the expected theoretical values $(\approx 0.125$ ns and $1 / 3$, respectively).

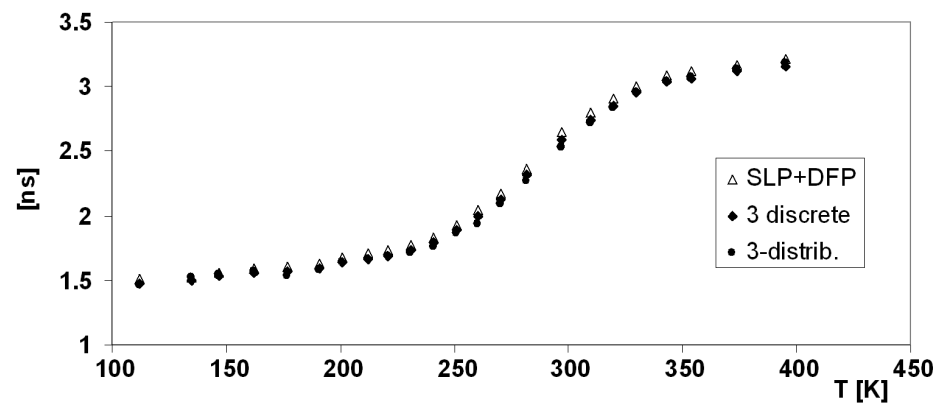

Fig. 2. The Ps pick-off lifetime determined from the three-discrete-component, threedistributed-component and SLP+DFP analyses.

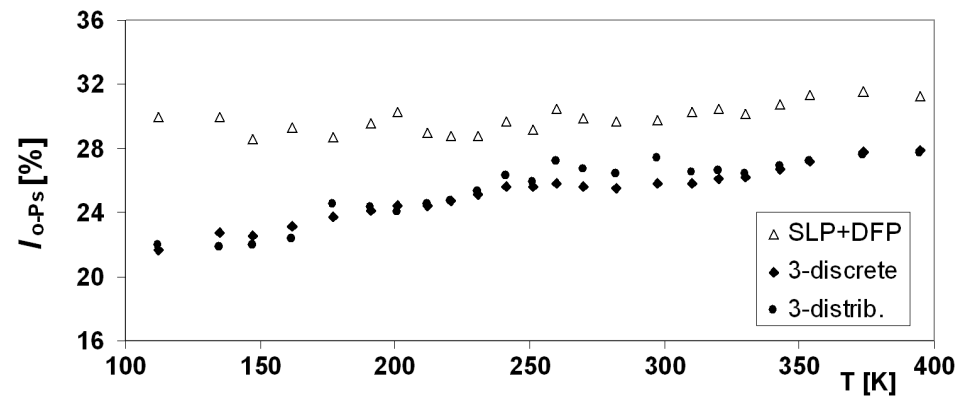

Fig. 3. The fraction of $o$-Ps determined from the three-discrete-component, threedistributed-component and SLP+DFP analysis.

determine the first two momenta of $\alpha_{i}(\lambda)$ only, i.e. the mean value of $\lambda$ and its standard deviation. Therefore in LT program $\alpha_{i}(\lambda)$ is introduced arbitrarily as the log-normal distribution 


$$
\alpha_{i}(\lambda) \mathrm{d} \lambda=\frac{1}{\sigma_{i}^{*}(2 \pi)^{1 / 2}} \exp \left(-\frac{\left(\ln \lambda / \lambda_{i 0}\right)^{2}}{2 \sigma_{i}^{* 2}}\right) \frac{\mathrm{d} \lambda}{\lambda},
$$

which is defined for positive values of $\lambda$ exclusively, and has maximum at $\lambda_{i 0}$ and width (on logarithmic scale) of about $2 \sigma_{i}^{*}$. The mean lifetime resulting from $\alpha_{i}(\lambda)$ is $\tau_{i}=\lambda_{i 0}^{-1} \exp \left(\sigma_{i}^{* 2} / 2\right)$, whereas dispersion of lifetimes from this mean value is $\sigma_{i}=\lambda_{i 0}^{-1}\left[\exp \left(\sigma_{i}^{* 2}\right)-1\right]^{1 / 2}$. The model (2) was fitted to the experimental data with 6 free parameters, $\tau_{1}, \tau_{2}, \sigma_{2}, I_{3}, \tau_{3}$, and $\sigma_{3}$.

The temperature dependences of some of the found parameters are shown in Fig. 4. Determined values of $\tau_{3}$ change very similar to those obtained from the three-discrete-component analysis (Figs. 4, 2). The dispersion $\sigma_{3}$, in the temperature range from 112 to $320 \mathrm{~K}$, seems to follow the changes of $\tau_{3}$. Above $320 \mathrm{~K}$ it suddenly declines. Similar declination of $\sigma_{3}$ was observed also for an epoxy resin [11]. However, usually $\sigma_{3}$ at $T_{\mathrm{k}}$ (or just below $T_{\mathrm{k}}$ ) becomes a weakly increasing temperature function [7-10, 12-15].

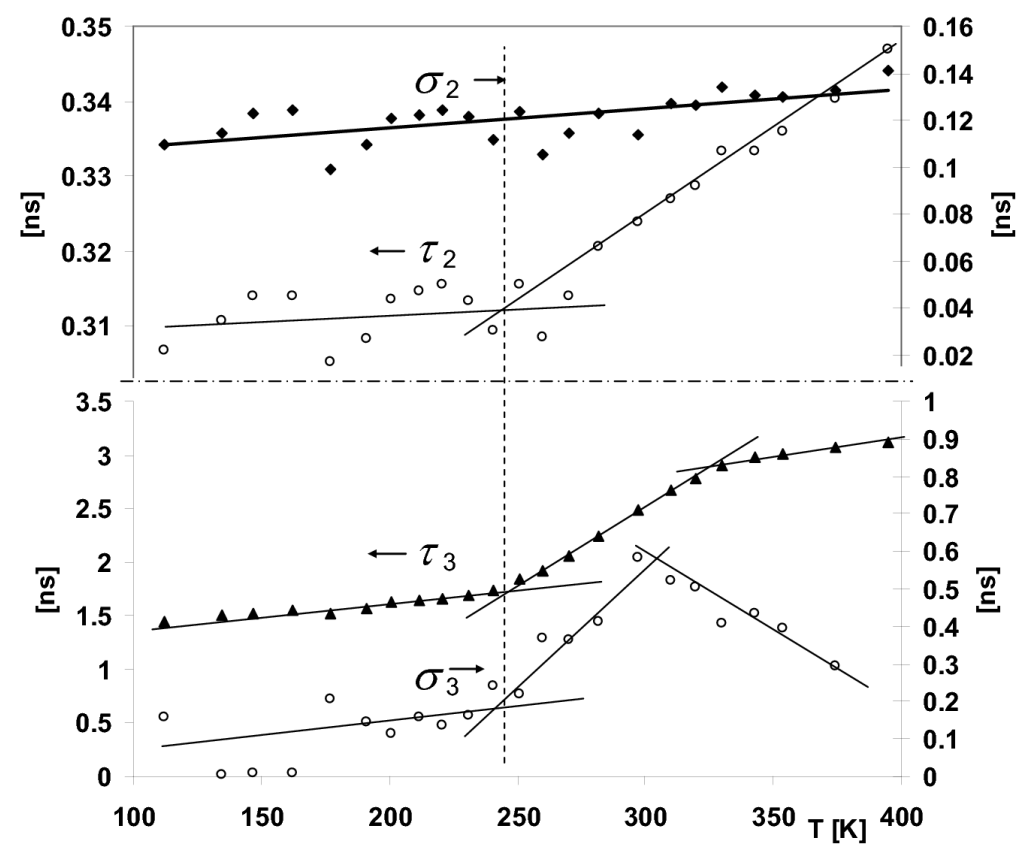

Fig. 4. Some parameters determined with the three-distributed-component analysis (from top) $\tau_{2}$ - the mean lifetime of $\mathrm{e}^{+}, \sigma_{2}$ - dispersion of lifetimes from $\tau_{2}, \tau_{3}$ the mean lifetime of $o$-Ps and $\sigma_{3}$ - dispersion of lifetimes from $\tau_{3}$. The vertical line indicates the glass temperature in polyethylene determined from the $\tau_{3}$ temperature dependence.

Values of $\tau_{2}$ from $112 \mathrm{~K}$ up to $T_{\mathrm{g}}$ seems to be constant (Fig. 4 ). Above $T_{\mathrm{g}}$ they increase monotonically from about $0.31 \mathrm{~ns}$ to $0.35 \mathrm{~ns}$, whereas $\sigma_{2}$ is almost constant 
and fluctuates around $0.13 \mathrm{~ns}$. The $p$-Ps lifetime $\tau_{1}$ (not showed) decreases slightly from 0.13 to $0.09 \mathrm{~ns}$ with large scatter of $\approx 0.01 \mathrm{~ns}$. The temperature dependence of $I_{3}$, determined from the three-distributed-component analysis, is similar to that obtained from the three-discrete-component analysis (Fig. 3), in spite of the fact that now $I_{1}$ satisfies the constraint $I_{1} / I_{3}=1 / 3$.

\subsection{Analysis with the combined SLP and DFP models}

In this analysis combined SLP (slow localization of Ps) and DFP (delayed formation of Ps) models were used [16-18].

SLP is based on following assumptions: A positron injected into polymer takes part in fast processes inside the positron spur and then leaves the terminal spur (blob) as a free particle or as a loosely bound pair $\mathrm{e}^{+}-\mathrm{e}^{-}[24]$. Because of the spin exchange repulsion between the electron of pair $\mathrm{e}^{+}-\mathrm{e}^{-}$and the core electrons, the pair is pushed out, into the holes of free volume of larger and larger size. In this way, de-excitation of the pair becomes lower providing a gradual transformation of the pair into a stable Ps and its final localization in the elementary free volume.

If a free positron leaves the blob, it diffuses through the material and finally annihilates from the "free" or a trapped state, or forms a positronium after meeting an electron trapped in a shallow potential well. This is a basis of DFP model.

We described the slow relaxation and localization of Ps by the following empirical formula:

$$
\lambda_{o / p}(t)=\lambda_{o / p i n t r}(t)+\lambda_{p-o}(t)
$$

where $\lambda_{o / p}(t)$ is the time dependence of the annihilation rate of $o$-Ps $/ p$-Ps and

$$
\lambda_{o / p \text { intr }}(t)=\lambda_{o / p \text { intr }}(\infty)\left[1-\exp \left(-t / \tau_{\text {relax }}\right)\right]
$$

represents changes of its intrinsic annihilation rate whereas

$$
\lambda_{p-o}(t)=\lambda(\infty)+[\lambda(0)-\lambda(\infty)] \exp \left(-t / \tau_{\text {local }}\right)
$$

describes the changes in its pick-off annihilation rate caused by the slow localization. $\lambda_{o / p \text { intr }}(\infty)$ denotes the $o$-Ps $/ p$-Ps intrinsic lifetime in its stable ground state $\left(1 / 142\right.$ or $1 / 0.125 \mathrm{~ns}$, respectively), $\tau_{\text {relax }}$ is the time of internal relaxation of Ps, whereas $\lambda(\infty)=1 / \tau(\infty)$ is the asymptotic (after Ps localization in the free volume) pick-off annihilation rate (reciprocal of respective pick-off lifetime), $\lambda(0)$ is the pick-off annihilation rate of the loosely bound $\mathrm{e}^{+}-\mathrm{e}^{-}$pair at $t=0$ (assumed equal to the annihilation rate of free $\mathrm{e}^{+}$, i.e. $\left.\lambda(0)=\lambda_{+}=1 / \tau_{+}\right)$, and $\tau_{\text {local }}$ is the time needed for Ps localization.

The model function which describes a part of lifetime spectrum originating from Ps annihilations during the SLP process is a function of four parameters $\tau(\infty), \tau_{+}, \tau_{\text {relax }}, \tau_{\text {local }}$. The function makes use from the assumption that the relative intensities related to $p$-Ps and $o$-Ps are as 1:3. Exact form of the function is given anywhere [16-18].

The fate of a positron which left the blob as a free particle is described by a set of kinetic equations 


$$
\begin{aligned}
\frac{\mathrm{d} c_{+}}{\mathrm{d} t} & =-\lambda_{+} c_{+}-\mu c_{+}-\kappa c_{+}, \\
\frac{\mathrm{d} c_{+}^{t}}{\mathrm{~d} t} & =-\lambda_{+}^{t} c_{+}^{t}+\mu c_{+}, \\
\frac{\mathrm{d} c_{p}}{\mathrm{~d} t} & =-\lambda_{p} c_{p}+\frac{1}{4} \kappa c_{+} \\
\frac{\mathrm{d} c_{o}}{\mathrm{~d} t} & =-\lambda_{o} c_{o}+\frac{3}{4} \kappa c_{+}
\end{aligned}
$$

The $c_{+}$and $c_{+}^{\mathrm{t}}$ parameters denote probabilities of the positron annihilation from the free or trapped states, $\tau_{+}^{\mathrm{t}}=1 / \lambda_{+}^{\mathrm{t}}$ is the positron lifetime in the trapped state, $c_{o}$ and $c_{p}$ are probabilities of annihilation of the $o$-Ps or $p$-Ps produced in the process of delayed Ps formation. $\mu$ is the positron trapping rate and $\kappa$ is the rate of the delayed Ps formation.

Finally, the model function, taking into account both SLP and DFP processes, is a function of eight independent parameters and time

$$
s(t)=s\left[I_{\mathrm{blob}}, \tau(\infty), \tau_{+}, \tau_{\text {relax }}, \tau_{+}^{\mathrm{t}}, \mu, \kappa ; t\right] .
$$

$I_{\mathrm{blob}}$ denotes the fraction of positrons injected into sample which formed Ps during the blob reactions (i.e. the efficiency of Ps formation in the blob). The function $s(t)$ was fitted to the experimental spectra ${ }^{\dagger}$. To avoid too big scatter of the results the model parameter $\tau_{+}$was fitted with a free but common value to all the spectra measured at different temperatures and the same constraints were put on $\tau_{+}^{\mathrm{t}}$. The preliminary results suggested a linearly increase in $\tau_{\text {relax }}$ with temperature. Therefore in the final analysis $\tau_{\text {relax }}$ was constrained to satisfy the relation $\tau_{\text {relax }}=\tau_{\text {relax }}(0)(1+\alpha T)$. The coefficients $\alpha$ and $\tau_{\text {relax }}(0)$ had free but common values for the whole series of spectra. Obtained values of all the common parameters are shown in Table.

\section{TABLE}

Found values of parameters which were assumed independent of temperature.

\begin{tabular}{c|c|c|c}
\hline \hline$\tau_{+}[\mathrm{ns}]$ & $\tau_{+}^{\mathrm{t}}[\mathrm{ns}]$ & $\alpha[1 / \mathrm{K}]$ & $\tau_{\text {relax }}(0)[\mathrm{ns}]$ \\
\hline 0.339 & 0.676 & 0.00145 & 0.0935
\end{tabular}

Figure 5 shows the determined values of some temperature-dependent parameters from the SLP+DFP analysis. The Ps localization time $\tau_{\text {local }}$ decreases with elevated temperature from $0.19 \mathrm{~ns}$ at $112 \mathrm{~K}$ to $0.054 \mathrm{~ns}$ at $390 \mathrm{~K}$. The Ps localization time is quite long, which results from long Ps thermalization time. The thermalization process goes mainly through Ps-phonon interactions. The phonon energy requires a great number of collisions between Ps and the surrounding molecules, before Ps reaches the lowest energy. The decrease in $\tau_{\text {local }}$ with

\footnotetext{
${ }^{\dagger}$ For abbreviation we will denote the method of analysis which uses the SLP and DFP models by SLP+DFP.
} 


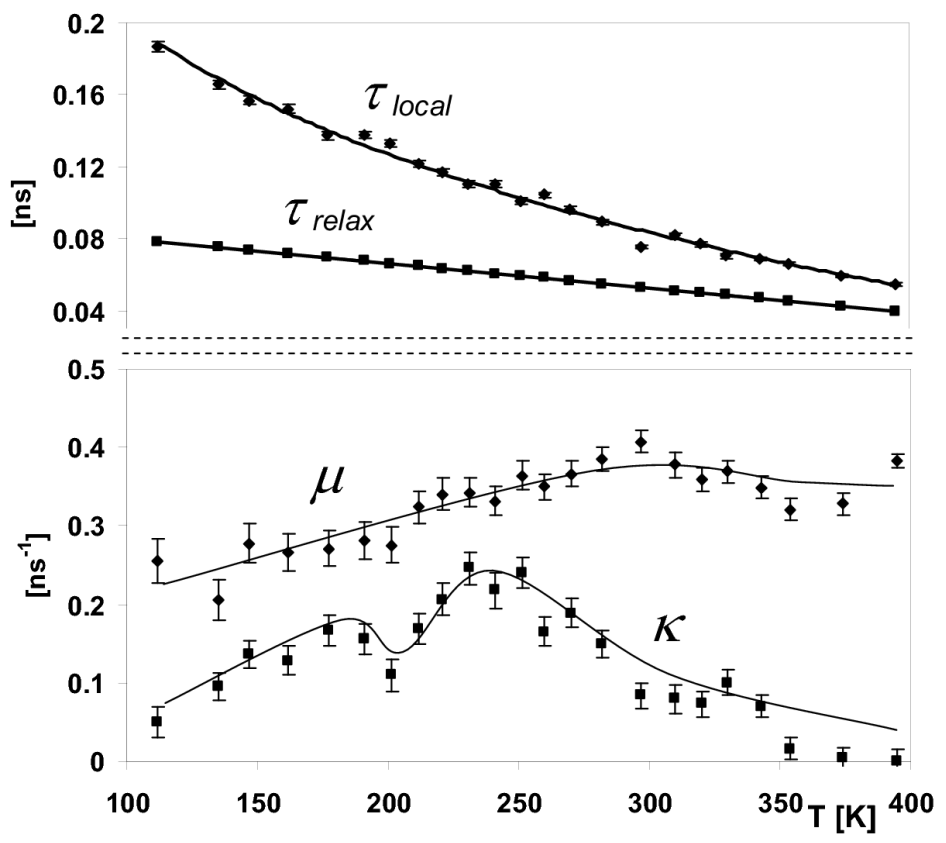

Fig. 5. Temperature dependences of the time of Ps localization $\left(\tau_{\text {local }}\right)$ in centers of free volume, the Ps relaxation time $\left(\tau_{\text {relax }}\right)$, the trapping rate $(\mu)$ of $\mathrm{e}^{+}$from the bulk, and the rate $(\kappa)$ of delayed formation of Ps from free $\mathrm{e}^{+}$and a trapped electron. The dependence of $\tau_{\text {relax }}$ was assumed linear.

temperature seems to be reasonable since at higher temperature the material becomes less "stiff" so a higher portion of Ps energy can be transferred to the heat bath during a collision of Ps with a molecule. The temperature changes of the Ps relaxation time $\left(\tau_{\text {local }}\right)$ follow the changes of $\tau_{\text {local }}$. It is understandable, since better localization of Ps in free volume causes smaller perturbation of the internal energy levels in Ps and enables the pair $\mathrm{e}^{+}-\mathrm{e}^{-}$better contact.

\subsection{Comparison of results of different models}

The pick-off time $\tau(\infty)$ of localized Ps in SLP + DFP has the same meaning as $\tau_{3}$ in the "conventional" three-component analyses of lifetime spectra ${ }^{\ddagger}$. Indeed, Fig. 2 shows the temperature dependence of $\tau(\infty)$ and $\tau_{3}(T)$ obtained from the three-discrete-component and three-distributed-component analyses. One can see a very good agreement between the parameters determined by those three different models.

Such agreement is not observed for the relative intensity of $o$-Ps (Fig. 3). In the three-component models the intensity is represented by $I_{3}$. In SLP+DFP the intensity is $I_{o-\mathrm{Ps}}=\frac{3}{4}\left(I_{\mathrm{blob}}+I_{\mathrm{DFP}}\right)$. The second term in the sum relates to the

\footnotetext{
${ }^{\ddagger}$ Really, $1 / \tau_{3}$ should be equal to $1 / \tau(\infty)+1 / 142$.
} 
amount of Ps formed in the delayed Ps formation and, according to solutions of the set of Eqs. (5a) to (5d) can be calculated as [16-18]:

$$
I_{\mathrm{DFP}}=\frac{\left(1-I_{\text {blob }}\right) \kappa}{\lambda_{+}+\mu+\kappa} .
$$

In the whole range of temperatures, the determined values of $I_{o-P s}$ are higher than the respective values of $I_{3}$ from both three-component analyses (Fig. 3). It becomes clear, if one takes into account, that $I_{3}$ is the fraction of $o$-Ps annihilating from the fully localized state, whereas $I_{o-P s}$ includes also additional $o$-Ps annihilations from non-entirely localized states. The temperature dependences of $I_{o-P s}$ and $I_{3}$ are different. It can be understood on basis of SLP+DFP. According to $\mathrm{SLP}+\mathrm{DFP}, I_{o-\mathrm{Ps}}$ is the total efficiency of $o$-Ps formation in the blob and in the process of delayed Ps formation, whereas $I_{3}$ depends not only on this efficiency but also on the Ps localization and relaxation times.

The quality of fit, defined as the mean fit variance $\left(\chi^{2}\right)$ for all the spectra measured at different temperatures, were 1.106 for the three-discrete-component analysis, 1.043 for the three-distributed component analysis and 1.041 for the SLP+DFP. As it is seen, the three-distributed-component model and SLP+DFP fit best and with almost the same excellent quality.

A good quality of fit is not sufficient argument for validity of the theoretical model used in analysis. A confirmation of its validity requires additionally a good physical meaning and a self-consisting of results.

The main point of the three-distributed-component model is the assumption about almost immediate localization of Ps $\left(\right.$ and $\left.\mathrm{e}^{+}\right)$in a randomly chosen freevolume hole without a possibility of hopping to another hole. The assumption about Ps remaining in a particular hole during its entire lifetime bases on results of Baugher et al. [25] who considered the localization o-Ps in free-volume holes as a particular case of the Anderson localization [26]. The authors calculated probability of tunneling of a particle, localized in one potential well, to another potential well of the same depth. They conclude that although the probability of such tunneling is very high for two wells of exact the same width, it decreases almost to zero for only slightly unequal wells. On the one hand, the thesis of Baugher et al. seems to be confirmed by low values of Ps diffusion coefficients measured in polymers [27] but, on the other hand, it is in contradiction with an experimental result of work by Kansy and Suzuki [17] where it was shown that the long-lived part of polyethylene spectrum can be well fitted by a single exponential component. Moreover, the calculations of Baugher et al. took into account no interaction between the localized particle and the heat bath. Maybe the interaction could change the probability of the tunneling process to values estimated by $\mathrm{Yu}$ et al. [28]. Anyway the problem seems to be not solved sufficiently and still needs some theoretical and experimental verifications.

It is worthy to add that, in principle, the SLP+DFP model does not exclude a possibility of $o$-Ps lifetime dispersion due to its annihilation from the free-volume 
holes of different sizes. However, for all spectra analyzed up to now with SLP+DFP (papers [16-18] and the present one) there were no needs to take such a dispersion into account.

The combined SLP and DFP models described positronium in its early stages. Therefore the age-momentum-correlation (AMOC) spectroscopy could be a useful tool for verification the models. Unfortunately, there are only few results of AMOC for polymers in literature [29, 30]. In spite of this, authors of a recent paper [18] calculated, on basis of the SLP+DFP parameters derived from lifetime spectra for high density polyethylene, theoretical shapes of $S(t)$ curves of AMOC which should be observed for this polymer. The predicted shapes well agreed with the experimental ones measured by Suzuki et al. [29].

\section{Conclusion}

From the three considering models only the three-distributed-component model or SLP+DFP satisfy the theoretical predictions about the $p$-Ps lifetime and about the ratio of $p$-Ps and $o$-Ps intensities.

The three-distributed-component model well described the positron lifetime spectra of polymers but it seems to be valid only in this case when the thesis of the Anderson localization of positronium in the free volume is true. Additionally, the model assumes fast localization of Ps and $\mathrm{e}^{+}$.

The slow Ps localization and relaxation process combined with the process of delayed Ps formation well predict the shape of polymer lifetime spectra. This time the assumption of the Anderson localization is not necessary, although the model is not in contradiction with such assumption.

The problem of choosing a correct model of polymer lifetime spectrum requires, on the one hand, additional theoretical efforts and, on the other hand, an independent confirmation by other positron spectroscopies, especially by AMOC experiments.

\section{Acknowledgments}

Author is grateful to Dr. Giovanni Consolati for his agreement to use the experimental data of polyurethane in the present work.

\section{References}

[1] O.E. Mogensen, Positron Annihilation in Chemistry, Springer, Berlin 1995.

[2] N. Nakanishi, Y.C. Jean, in: Positron and Positronium Chemistry, Studies in Physical and Theoretical Chemistry, Vol. 57, Eds. D.M. Schrader, Y.C. Jean, Elsevier Sci. Publ., Amsterdam 1988, Ch. 2, p. 169.

[3] Y.C. Jean, Microchem. J. 42, 72 (1990).

[4] Y.C. Jean, Mater. Sci. Forum 175/178, 59 (1995).

[5] G. Dlubek, S. Eichler, Ch. Hbner, Ch. Nagel, Nucl. Instrum. Methods Phys. Res. B 149, 501 (1999). 
[6] G. Dlubek, S. Eichler, Ch. Hübner, Ch. Nagel, Phys. Status Solidi A 174, 313 (1999).

[7] G. Dlubek, A. Sen Gupta, J. Pionteck, R. Häßler, R. Krause-Rehberg, H. Kaspar, K.H. Lochhaas, Polymer 46, 6075 (2005).

[8] G. Dlubek, A. Sen Gupta, J. Wawrzyszczuk, D. Kilburn, J. Pionteck, R. KrauseRehberg, T. Goworek, M.A. Alam, H. Kaspar, K.H. Lochhaas, Acta Phys. Pol. A 107, 685 (2005).

[9] G. Dlubek, V. Bondarenko, I.Y. Al-Qaradawi, D. Kilburn, R. Krause-Rehberg, Macromol. Chem. Phys. 205, 512 (2004).

[10] G. Dlubek, U. De, J. Pionteck, N. Yu Arutyunov, M. Edelmann, R. KrauseRehberg, Macromol. Chem. Phys. 206, 827 (2005).

[11] G. Dlubek, E.M. Hassan, R. Krause-Rehberg, J. Pionteck, Phys. Rev. E 73, 031803 (2006).

[12] G. Dlubek, J. Non-Cryst. Solids 352, 2869 (2006).

[13] D. Bamford, G. Dlubek, T. Lüpke, D. Kilburn, J. Stejny, T.J. Menke, M.A. Alam, Macromol. Chem. Phys. 207, 492 (2006).

[14] D. Kilburn, J. Wawrzyszczuk, G. Dlubek, J. Pionteck, R. Häßler, M.A. Alam, Macromol. Chem. Phys. 207, 721 (2006).

[15] G. Dlubek, J. Pionteck, M.Q. Shaikh, E.M. Hassan, R. Krause-Rehberg, Phys. Rev. E 75, 021802 (2007).

[16] J. Kansy, T. Suzuki, Acta Phys. Pol. A 110, 593 (2006).

[17] J. Kansy, T. Suzuki, Radiat. Phys. Chem. 76, 291 (2007).

[18] J. Kansy, T. Suzuki, Radiat. Phys. Chem. 76, 759 (2007).

[19] G. Consolati, J. Kansy, M. Pegoraro, F. Quasso, L. Zanderighi, Polymer 39, 3491 (1998).

[20] J. Kansy, Nucl. Instrum. Methods Phys. Res. A 374, 235 (1966).

[21] C.L. Wang, T. Hirade, F.H.J. Maurer, M. Eldrup, N.J. Pedersen, J. Chem. Phys. 108, 4654 (1998).

[22] T. Hirade, F.H.J. Maurer, M. Eldrup, Radiat. Phys. Chem. 58, 465 (2000).

[23] Y. Ito, T. Hirade, E. Hamada, T. Suzuki, Y. Ito, Acta Phys. Pol. A 95, 533 (1999).

[24] S.V. Stepanov, V.M. Byakov, J. Chem. Phys. 116, 6178 (2002).

[25] A.H. Baugher, W.J. Kossler, K.G. Petzinger, R.H. Pater, Mater. Sci. Forum 255-257, 57 (1997).

[26] P.W. Anderson, Phys. Rev. 109, 1492 (1958).

[27] Y.C.Jean, Macromolecules 29, 5756 (1996).

[28] Z. Yu, J.D. McGervey, A.M. Jamieson, R. Simha, Macromolecules 68, 6268 (1995).

[29] N. Suzuki, T.Hirade, T.F. Saito, T. Hyodo, Radiat. Phys. Chem. 68, 647 (2003).

[30] C. Dauwe, B. Van Waeyenberge, J. De Baerdemaeker, Acta Phys. Pol. A 107, 623 (2005). 